\title{
Essais
}

ESSAIS

Revue interdisciplinaire d'Humanités

$15 \mid 2019$

Jouer l'histoire

\section{La Grande Guerre et les jeux vidéo. La recherche historique face à Battlefield 1}

Nicolas Patin

\section{OpenEdition}

1 Journals

Édition électronique

URL : http://journals.openedition.org/essais/1722

DOI : 10.4000/essais. 1722

ISSN : 2276-0970

Éditeur

École doctorale Montaigne Humanités

Édition imprimée

Date de publication : 15 octobre 2019

Pagination : 119-130

ISBN : 979-10-97024-07-9

ISSN : 2417-4211

\section{Référence électronique}

Nicolas Patin, "La Grande Guerre et les jeux vidéo. La recherche historique face à Battlefield 7 », Essais [En ligne], 15 | 2019, mis en ligne le 05 octobre 2020, consulté le 12 octobre 2020. URL : http:// journals.openedition.org/essais/1722 ; DOI : https://doi.org/10.4000/essais.1722 


\section{La Grande Guerre et les jeux vidéo. La recherche historique face à Battlefield 1}

\section{Nicolas Patin}

Une proportion toujours plus importante d'étudiants et d'étudiantes s'oriente vers l'histoire à l'université du fait d'une expérience vidéoludique ${ }^{1}$. Les écrans et les manettes ont en partie remplacé le film ou le livre, qui, un jour, avait créé ce déclic, celui de l'intérêt - si ce n'est de la passion - pour la discipline historique. Pourtant, cette dernière ne s'intéresse que très lentement à ces productions et à ses pratiques culturelles. Effet de génération, très certainement, mais effet de frilosité également. Les jeux vidéo sont encore entachés des clichés des années 1980-1990 autour d'une pratique divertissante - voire abêtissante - et presque uniquement masculine ${ }^{2}$. Lors d'un cycle autour de la question organisée pendant deux mois dans mon université, j'ai pu constater que parmi la grande majorité des collègues, la perception de l'univers vidéoludique se faisait, notamment chez les professeurs, par un seul biais : la pratique de leurs propres enfants. Ils sont très peu à avoir tenu une manette entre les mains. À l'heure où l'industrie du jeu vidéo est la première industrie culturelle mondiale ${ }^{3}$ et où la musique, le cinéma, la bande dessinée semblent avoir trouvé droit de cité dans certains espaces du monde de la recherche, il semble qu'un retard doit être rattrapé. Les Game Studies y contribuent ${ }^{4}$. Mais en histoire, il reste encore un long chemin à parcourir.

1 J'ai mené une rapide enquête auprès de mes étudiants de L1 et de L2 à l'Université Bordeaux Montaigne à partir de la plateforme Askabox, au mois d'avril 2017. Sur 100 étudiants visés, 50 ont répondu. $83 \%$ jouent à des jeux vidéo ; $75 \%$ jouent au moins une fois par semaine ; $90 \%$ jouent à des jeux vidéo à caractère historique. Ces chiffres sont évidemment impressionnistes, mais tout de même intéressants.

2 Fanny Lignon (éd.), Genre et jeux vidéo, Toulouse, Presses universitaires du Midi, 2015.

3 Naomi Alderman, "How videogames took over the world ", theguardian.com, 22 décembre 2009. Les jeux vidéo ont "réclamé leur couronne de plus grande industrie du divertissement au monde ».

4 Benjamin Beil, Thomas Hensel, Andreas Rauscher (Hrsg.), Game Studies, Wiesbaden, Springer VS, 2018. 
C'est d'autant plus dommageable que très souvent, ce sont les jeux vidéo traitant de telles ou telles périodes historiques qui sont susceptibles, aujourd'hui, de déclencher des débats sur les aspects d'une époque. Les jeux de guerre et FPS 5 , en particulier, sont les premiers à tomber sous le feu de la critique, mais une critique qui se fait encore largement en dehors du monde universitaire. Si l'on s'intéresse sommairement à la morphologie des débats autour des séries Call of Duty (Infinity Ward / Activision), Battlefield (DICE / Electronic Arts) ou la bien connue Assassin's Creed (Ubisoft), il est étonnant de constater que la recherche académique est presque totalement absente. Pour des dizaines d'articles publiés dans les revues spécialisées sur les jeux vidéo, pour des dizaines et des centaines de pages de débats enflammés sur les forums ${ }^{6}$, il n'émerge - et seulement récemment - que quelques avis épars de spécialistes reconnus dans un média de grande envergure, Le Monde faisant ici office de précurseur ${ }^{7}$. Ce qu'il est particulièrement intéressant de constater, c'est la manière d'appréhender l'objet de chaque côté de cette frontière imperméable : très souvent, là encore si l'on veut en faire un résumé sommaire, le sujet débattu par les érudits-gamers est toujours le même : le réalisme du jeu, sa véracité historique. Mais cette véracité prend toujours, peu ou prou, le même chemin : celui d'une histoire matérielle, d'une histoire militaire et d'une histoire des techniques. Les discussions que j'ai pu avoir avec mes étudiants tournent souvent autour des mêmes aspects, surtout pour les jeux de guerre : pourquoi telle arme n'est-elle pas respectée ? Pourquoi utiliser tel uniforme? La stratégie de telle bataille est totalement irréaliste... Des questions auxquelles, en tant qu'historien du politique, du social ou du culturel, je n'ai jamais été, à aucune occasion, capable de répondre. Inversement, des questions qui me paraissent totalement légitimes - la manière dont les structures politiques, les interactions sociales ou la vie quotidienne sont dépeintes - intéressent souvent peu mes étudiants, bien que ce ne soit pas la totalité. Se joue ici un conflit très intéressant, qui mériterait d'être étudié à part entière par la sociologie : comment ces jeunes joueurs, ayant accumulé un capital culturel considérable, ne peuvent-ils le mettre en valeur, car l'institution dans laquelle ils essayent de

5 First-person shooter ou " jeu de tir à la première personne ». Voir notamment Carl Therrien, "Inspecting Video Games Historiography Through Critical Lens: Etymology of the FirstPerson Shooter Genre ", in Game Studies, the international journal of computer game research, volume 15, issue 2, décembre 2015.

6 Un exemple sur Reddit : "How Historically accurate is Battlefield 1? ». Voir : https://www. reddit.com/r/history/comments/57f3u6/how_historically_accurate_is_battlefield_1/.

7 Voir les nombreux articles que le journal Le Monde a par exemple consacrés à la sortie du jeu Assassin's Creed Origins (Ubisoft, 2017). Voir Damine Leloup, "On a testé... "Assassin's Creed Origins", morts sur le Nil », 27 octobre 2017 ; William Audureau, «"Assassin's Creed Origins" : ce qui est fidèle à l'Égypte antique, ce qui ne l'est pas ", 27 octobre 2017 ; ou plus récemment, pour la sortie du dernier opus : William Andureau, "Assassin’s Creed Odyssey": une carte postale de la Grèce, pas un cours d'histoire », 5 octobre 2018. 
le faire fonctionne comme un champ aux codes différents, et ne cache d'ailleurs souvent pas son mépris pour ces érudits qui en savent parfois beaucoup plus qu'il n'y paraît.

Dans le cadre du Centenaire de 1914-1918, la Mission du Centenaire ${ }^{8}$ - l'institution visant à coordonner les activités de commémorations du conflit en France - a pris en compte ces enjeux, s'impliquant massivement dans le développement du jeu Soldats Inconnus : Mémoires de la Grande Guerre (Ubisoft) ${ }^{9}$. Cette innovation a globalement été un succès. Mais en face du petit village gaulois - le jeu a été développé à Montpellier - c'est bien le blockbuster Battlefield 1 (DICE/Electronic Arts, 2016) qui a été l'événement historico-ludique du Centenaire, avec plus de vingt millions de joueurs dans le monde ${ }^{10}$. Quelques débats avec des historiens ont eu lieu çà et là ${ }^{11}$. La principale critique a été l'absence remarquée, dans la campagne solo ${ }^{12}$, des armées françaises et russes, ou le caractère parfois fantaisiste de tel épisode de la guerre, notamment la libération d'Amiens, se déroulant comme une guérilla urbaine anachronique. Très souvent, des historiens sont convoqués par des médias spécialisés pour répondre à telle ou telle question technique - et légitime mais il n'y a pas eu d'analyse de fonds du jeu comme produit culturel participant de la mémoire de la Guerre. Je vais donc essayer de proposer des pistes pour cette analyse, en tant qu'historien spécialiste de la Grande Guerre dans son versant allemand et en tant que joueur - très épisodique, dois-je confesser - mais ayant eu l'occasion de passer une vingtaine d'heures sur le mode " campagne solo » du jeu Battlefield 1 (je ne prendrais pas en compte le mode multijoueurs). Il y aurait une absurdité à essayer d'appliquer à ce jeu ce que Jean Norton Cru a appliqué aux témoignages de la Grande Guerre, une sorte de filtrage radical du véritable et du véridique ${ }^{13}$. Je vais plutôt essayer d'ana-

8 Voir le site institutionnel : http://centenaire.org/fr/la-mission/la-mission-du-centenaire.

9 Voir notamment : http://centenaire.org/fr/autour-de-la-grande-guerre/jeu-video/reportage/ autour-du-jeu-video-soldats-inconnus-le-carnet-de-1.

10 Eddie Makuch, "Battlefield 1 Hits 21 Million Players ", Gamespot.com, 28 juillet 2017 : https://www.gamespot.com/articles/battlefield-1-hits-21-million-players/1100-6452101/.

11 Wes Fenlon, "We showed Battlefield 1 to a World War I historian ", 20 juillet 2016 : https:// www.pcgamer.com/we-showed-battlefield-1-to-a-world-war-i-historian/. Voir également : Stéphanie Trouillard, «Battlefield 1 n’est pas un jeu sur la Première Guerre mondiale », France24. com, 5 novembre 2016, https://www.france24.com/fr/20161105-battlefield-1-jeu-videopremiere-guerre-mondiale-critique-historien-test-gameplay ; Peter Bathge, "Battlefield 1 : "Die Einzelspieler-Kampagne ist eine einziges Lügenmärchen - wunderschön und hirnrissig" ", Pcgames.de, 3 novembre 2016 : http://www.pcgames.de/Battlefield-1-2016-Spiel-54981/ Specials/Singleplayer-Solo-Kampagne-Kolumne-Kommentar-Luegenmaerchen-1210949/.

12 Une campagne est la partie scénarisée d'un jeu, souvent jouée en solo. Elle se construit autour de missions qui s'enchaînent.

13 Jean Norton Cru, Témoins, essai d'analyse et de critique des souvenirs de combattants édités en français de 1915 à 1928, Nancy, Presses universitaires de Nancy, 2006 [1929]. Pour des analyses sur ce travail fondateur, voir Nicolas Beaupré, Écrire en guerre, écrire la guerre. France, Allemagne 1914-1920, Paris, CNRS Éditions, 2006, et Charlotte Lacoste, Séductions du bourreau. Négation des victimes, Paris, Puf, 2010. 
lyser la manière dont ce jeu - qui n’a bénéficié que d'un conseil historique sommaire $^{14}$ - résonne avec certaines tendances de l'historiographie contemporaine, dépeint certaines choses avec un grand talent, tout comme il est contraint, pour des raisons techniques liées à la « jouabilité » (gameplay $\left.{ }^{15}\right)$, de faire de grosses erreurs historiques. N'étant pas spécialiste des Game studies, je revendique une vision extérieure au champ, celle d'un historien traditionnel, et je remercie donc mes relecteurs pour m'avoir conseillé telle ou telle référence plus spécialisée dans ce domaine. Je m’arrêterais sur trois thématiques : le caractère international de la guerre ; la représentation des relations entre soldats et enfin, les formes de violence.

\section{Guerre mondiale, jeu mondial}

Le Centenaire de 1914-1918, avec son flot ininterrompu de publications, de colloques et de conférences a montré l'évidence : la Grande Guerre a été une guerre européenne et plus largement, mondiale. Que le théâtre des opérations se soit enraciné profondément en Europe, et notamment en France, ne change rien à la diffusion du conflit, à l'Est, évidemment, ce front dont Winston Churchill considérait qu'il avait été " oublié ${ }^{16}$ ou en Orient. La présence des milliers de soldats coloniaux venus du monde entier, des empires britanniques et français, ne faisait qu'accentuer le caractère international de la conflagration. Et ce n'est que récemment - d'autant plus avec le Centenaire que les chercheurs sont parvenus à décloisonner des historiographies souvent très nationales pour prendre en compte l'immense hybridation culturelle qu'a représentée la Grande Guerre ${ }^{17}$. De ce point de vue, Battlefield 1, qui vise autant des objectifs commerciaux de séduction de divers marchés nationaux et linguistiques qu'une véracité historique, offre une expérience de jeu historique tout à fait satisfaisante.

14 Les crédits du jeu, qui durent 16 minutes, n’indiquent en tout état de cause pas de conseils historiques en propre, par des historiens universitaires, ce qui ne signifie pourtant pas qu'aucun travail de conseil n'a eu lieu.

15 Le terme " gameplay ", que je traduis sans originalité par "jouabilité ", est en réalité plus complexe. En effet, " jouabilité " renvoie au terme playability, qui est une transposition du concept d'usabilité (usability) dans le jeu vidéo. On peut considérer le "gameplay " comme lié «aux interactions possibles entre le représentant virtuel du joueur (avatar) et les éléments constituant le monde numérique ". Voir Charles-Érick Bélanger-Gagnon, L'Induction du sentiment de présence dans le jeu vidéo, Mémoire présenté à la Faculté des études supérieures de l'Université Laval dans le cadre du programme de maîtrise sur mesure en Design, animation et cyberculture pour l'obtention du grade de maitre ès arts (M. A.), Québec, Université Laval, 2010, p. 16-17.

16 Winston Churchill, The World Crisis, volume V. The Unknown War. The Eastern Front, Charles Scribner's Sons, 1931.

17 On peut penser au colloque tenu en septembre 2013 à la Cité de l'immigration et à l'Institut historique allemand, "Altérités en guerre : la Grande Guerre comme moment interculturel ». 
La première mission - une fois sortie de l'introduction-tutoriel sur laquelle nous reviendrons - met aux prises des soldats britanniques - Townsend, MacManus, Edwards et Finch - manoeuvrant un char britannique Mark $V$ face aux lignes allemandes ${ }^{18}$. Elle s'intitule "Dans la boue et le sang " et son action se déroule à Ribécourt-la-Tour près de Cambrai à l'automne 1918. L'ambiance sonore participe d'une immersion internationale, les soldats allemands, à mesure que l'on s'en approche, s'exprimant dans leur propre langue, même si c'est pour dire des phrases relativement simplistes ${ }^{19}$. La mission se poursuit à Bourlon pour se terminer dans la ville de Cambrai. La deuxième mission, intitulée "Des amis haut placés ", se fonde sur le voyage d'un aviateur du Royal Flying Corps britannique, Clyde Blackburn, qui part des Alpes pour survoler la France et terminer à Londres. La troisième mission se déroule sur les lignes italiennes, auprès des unités d'élite (les Arditi) et est intitulée «Avanti Savoia!». La quatrième mission, intitulée "l'estafette ", narre la bataille de Gallipoli, vue depuis le point de vue des forces australiennes et néo-zélandaises de l'ANZAC dans cette défaite face à l'Empire ottoman. Enfin, la cinquième et dernière mission, "Rien n'est écrit ", se déroule au Moyen-Orient, autour du personnage de Lawrence d'Arabie décrit comme un "officier britannique peu orthodoxe ». Le message délivré par le jeu est clair : "La Grande Guerre couvre le monde entier».

On l'a dit, il a été reproché au jeu, par certains joueurs, de ne pas pouvoir incarner, au moins dans la campagne solo, des soldats français ou russes. On peut voir dans cette revendication aussi bien l'importance de la mémoire française de la Grande Guerre et un certain chauvinisme, tout comme un transfert de l'intérêt porté aux troupes soviétiques dans les jeux de Seconde Guerre mondiale sur le front Est de 1914-1918, peu connu. Ceci dit, pour l'historien qui s'empare de la manette, la première impression est plutôt satisfaisante, même si la guerre est globalement perçue du point de vue anglo-saxon. Il est très rare qu'un livre d'histoire aborde un kaléidoscope aussi large de situations guerrières et de lignes de front. Les combats italiens, notamment, sont très souvent portion congrue dans les récits de Première Guerre mondiale, dans les combats qui ont pu les opposer aux troupes austro-hongroises.

18 Pour toute personne qui ne possède - et ne veut pas posséder de console de jeu ou d'ordinateur de jeu - il est possible de regarder les campagnes, sur la plateforme de vidéos en ligne Youtube. Ces vidéos de gameplay sont très populaires. Une parmi d'autres : https://www.youtube.com/ watch?v=gCuYnREWeR0\&frags=pl\%2Cwn. Ceci dit, du point de vue méthodologique, si ces vidéos peuvent se substituer à une expérience de jeu pour l'analyse des parties filmées, l'expérience vidéoludique en elle-même est nécessaire pour réaliser une réelle objectivation.

19 Quelques exemples parmi d'autres : " Eh, camarade, tout va bien ? "; " Il faut que je boive un coup »; " Merde, il fait tellement froid». 


\section{Consentements, contraintes et camaraderie}

On le sait, les débats dans l'historiographie française de la Grande Guerre ont été nombreux et parfois virulents, pour répondre à la question de la ténacité combattante ${ }^{20}$. Très souvent caricaturées, deux " écoles ", celle de la " contrainte » et celle du " consentement » étaient censées résumer l'ensemble des positions possibles au sein du débat, notamment pour répondre à la question de la fabrication de l'obéissance dans les tranchées et à l'arrière. Ces débats, centrés sur une histoire sociale et culturelle du fait guerrier, invitent à scruter le jeu Battlefield 1 avec les outils des vingt dernières années de l'historiographie française. Et là encore, le jeu fait preuve d'une certaine maestria, donnant à voir des configurations plus complexes qu'il n'y parait. Prenons deux exemples précis.

Dans la première mission, les quatre protagonistes qui actionnent le char britannique semblent correspondre à des archétypes. Nous incarnons, en tant que joueurs, la jeune recrue Edwards. Il est chauffeur dans le civil, du moins est-ce son activité après la guerre et celle dont il s'acquitte pendant la mission : piloter le char et le réparer. Townsend, plus âgé, est la figure du chef, qui alterne encouragements paternalistes et ordres plus laconiques et directs ${ }^{21}$, figurant une autorité qui ne se contente pas d'une pure relation de contrainte. Comme l'a montré Emmanuel Saint-Fuscien dans son ouvrage $\grave{A}$ vos ordres ?, aucune forme d'obéissance n'aurait pu être obtenue durant les quatre ans et demi de guerre en ne recourant qu'à la dureté et la violence ${ }^{22}$. Toute autorité est, finalement, négociée et Townsend semble incarner cette forme de souplesse. Le premier échange des deux personnages est intéressant : la jeune recrue lance : " Je suis prêt à me battre pour ma patrie ${ }^{23}$ ", ce à quoi son supérieur répond : "Tant que vous savez reconnaître la marche avant de la marche arrière, moi, ça me va ». Il y va même d'une certaine dé-hiérarchisation en jouant la connivence virile avec les hommes de sa petite unité, en dépeignant le tank «Big Bess ", sous les traits d'une femme, celle "de vos rêves ". Le soldat MacManus incarne, quant à lui, une figure négative. Tout le temps en train de pester, il proteste contre les ordres et contre la guerre en elle-même. On apprend plus tard qu'il est un soldat aguerri, ayant des dizaines de missions à son actif. Il n’hésite pas à s'opposer de front à Townsend ("Vous êtes fous ? Vous allez nous

20 Antoine Prost, Jay Winter, Penser la Grande Guerre. Un essai d'historiographie, Paris, Le Seuil, 2004, p. 140 et suivantes.

21 À un moment de difficulté dans le jeu, il lâche à Edwards : «Edwards! Lâcher le pigeon, c'est un ordre, mon garçon, vous comprenez ce que je dis?".

22 Emmanuel Saint-Fuscien, À vos ordres. La relation d'autorité dans l'armée française de la Grande Guerre, Paris, EHESS Éditions, 2011, p. 261-262.

23 Les dialogues sont extraits des sous-titres de la version française. Cette version ne rend évidemment pas toutes les subtilités du jeu, car Townsend, par exemple, a un très fort accent écossais dans la version originale. 
faire tuer ?»). Il pousse si loin ces rebuffades ("Et maintenant, qu'est-ce qui reste ? Des ordres aveugles... et le hasard ") que Townsend le remet à sa place (" Je ne veux plus vous entendre MacManus, c'est bien clair ? "). Reste un quatrième soldat, Finch, inquiet et fébrile, relativement jeune. Il tient un rôle important : il est tué dans un des premiers engagements du tank sous le feu ennemi. Edwards, qui est sorti de l'engin, le voit mourir sous ses yeux. La scène pourrait paraître anodine - et elle est, narrativement, relativement exagérée, puisque Edwards semble montrer beaucoup d'empathie et de souffrance face à la mort de son camarade (il crie "Finch est mort ") alors qu'il ne le connaît que depuis quinze minutes, mais elle dit quelque chose d'essentiel d'un des plus grands moteurs de la guerre, cette solidarité des tranchées que certains ont appelée, en la politisant, la " communauté du front ». C'était un des ressorts du consentement les plus puissants : les soldats continuaient souvent de se battre pour honorer la mémoire d'un ou plusieurs camarades tombés avant eux, sans nécessairement se sentir impliqués dans des formes plus institutionnalisées d'adhésion. La littérature de guerre fourmille de ces exemples ${ }^{24}$.

MacManus - une fois Finch tué - fonctionne comme le miroir d'Edwards : l'un est un jeune soldat à la fibre patriotique encore bien chevillée ${ }^{25}$; l'autre un vieux roublard, fatigué et proche du refus de guerre ${ }^{26}$. Alors que Townsend est blessé et le tank immobilisé, MacManus déserte, abandonnant Edwards à son sort. "C'est foutu ", lâche-t-il, « tout le monde s'est fait avoir ». Edwards le tance. MacManus s'emporte : "Je vous interdis de me parler comme ça. J'ai plus de missions à mon actif que vous n'en aurez jamais ! ", crie-t-il, avant de disparaitre. D'une certaine manière, ce dialogue bref représente une forme de confrontation entre les moments d'enthousiasme et d'inconscience de 1914 contre les refus de guerre et les rébellions de 1917. Happy end oblige, MacManus revient au dernier moment et sauve Edwards qui manque de se faire tuer. "Je crois que j'ai fait fausse route », lâche-t-il. Si la narration n'est pas spécialement ambitieuse, et ne fait qu'entrecouper les moments de gameplay sans réellement laisser le temps de s'attacher aux personnages, les représentations de l'autorité, du patriotisme et des refus de guerre tiennent compte de la diversité des positionnements dans la guerre. La séquence d'ouverture se termine même sur une fraternisation entre deux soldats ennemis.

On retrouve cette finesse dans la représentation des relations entre soldats aguerris et jeunes recrues, que ce soit dans cet exemple, ou dans la mission concernant l'ANZAC. Là encore, le jeu rejoint des éléments que l'on trouve

24 On peut la voir dans le rapport entre la jeune recrue Paul Bäumer et le soldat expérimenté Kat dans Erich Maria Remarque, À l'Ouest rien de nouveau, traduit de l'allemand par Alzir Hella et Olivier Bournac, Paris, Stock, 1957 [1928]. Notamment le chapitre III, p. 35-47. Voir également p. 56-57.

25 À l'arrière-plan sonore, au début du jeu, on entend un soldat crier : « Pour le roi et la patrie ! ».

26 Voir André Loez, 14-18. Les refus de la guerre. Une histoire des mutins, Paris, Gallimard, 2010. 
dans la littérature de Première Guerre mondiale ou dans l'historiographie. Un vieux militaire, Frederick Bishop, désigné comme un véritable héros dans son pays natal, l'Australie, est rejoint par une très jeune estafette, Jack Foster. Celui-ci s'est engagé volontairement, en mentant sur son âge qui est en dessous des normes de recrutement, pour se battre au côté de son héros. Il est reçu froidement par ce dernier, soldat expérimenté douchant, là encore, l'enthousiasme patriotique du va-t-en-guerre. Après une première mission, il le met face à des monceaux de cadavres, en criant « Regarde ça! Petit... Tes mensonges t'ont mené en enfer !» Face à ce baptême du feu très particulier, Jack vomit. Il est intéressant de constater que l'image donnée de la guerre n'a rien d'un simple récit centré sur l'héroïsme guerrier, mais que le jeu se fonde sur une représentation post-héroïque : les soldats ne sont pas courageux de facto, en défendant les valeurs chevaleresques de l'action de bravoure individuelle ; ils le deviennent une fois qu'ils ont enduré l'absurdité de la guerre industrielle ${ }^{27}$. Cela est d'autant plus visible dans la peinture qui est faite, dans la deuxième mission, du personnage de l'aviateur. Celui-ci, Clyde Blackburn, est un jeune pilote britannique, roublard, joueur, menteur et toujours prêt à se débiner. Dans une narration énergique, on le voit tour à tour tenter de sauver son coéquipier, hésiter à l'abandonner, risquer l'impossible au mépris des risques... en bref, incarner une forme d'héroïsme proche des romans de cape et d'épée du XIX ${ }^{\mathrm{e}}$ siècle. La fin de cet épisode est d'ailleurs totalement irréaliste : Blackburn combat à mains nues à trois cents mètres d'altitude, juché sur un dirigeable en flamme au-dessus de la Tamise. Il tombe et réussit tout de même à survivre. Il apostrophe alors le joueur pour terminer son histoire : "Mais en temps de guerre, les faits se mélangent un peu et vous entendrez sûrement d'autres versions... " Il laisse ainsi peser un doute sur le pacte de lecture initial de cet épisode. Du point de vue historique, ce personnage est très bien senti : les aviateurs - les " as » - comme le Baron Rouge Manfred von Richthofen ou Georges Guynemer pour la France étaient représentés comme les dernières figures réellement héroïques, celles que la guerre anonyme des tranchées n'avait pas encore éclaboussées dans leur superbe. Ces chevaliers du ciel incarnaient - par leur jeunesse et leur témérité - une forme d'aristocratie du combat, jeune et intrépide, connaissant un taux de mortalité extrêmement élevé à ce stade embryonnaire du développement technique de l'aviation ${ }^{28}$. De ce point de vue, et en dehors des informations d'histoire militaire sur l'utilisation de telle arme ou de tel biplan dans le jeu, le personnage complète une galerie qui - tout en étant celle d'une ouvre de fiction -

27 Arndt Weinrich, Der Weltkrieg als Erzieher. Jugend zwischen Weimarer Republik und Nationalsozialismus, Klartext, Essen, 2012, p. 65-68.

28 Bernd Hüppauf, "L'aviateur : preux chevalier ou héros moderne ? ", in Nicolas Beaupré, Gerd Krumeich, Nicolas Patin et Arndt Weinrich (éds), La Grande Guerre vue d'en face. 19141918. Nachbarn im Krieg, Paris, Albin Michel, 2016, p. 96-101. 
donne une représentation relativement fidèle d'archétype de soldats que l'on retrouve dans la littérature de témoignage ou dans les mémoires des soldats de la Grande Guerre.

\section{Les formes consacrées de la violence}

Le troisième aspect intéressant, et peut-être le plus central du jeu Battlefield 1, est la mise en scène de la violence. Le débat, là encore, a agité les différentes traditions historiographiques françaises, voire internationales. En effet, le récit historique des années 1960-1970 avait mis à l'honneur une écriture de la violence très anonyme des tranchées. Face à cette première guerre " industrielle et démocratique ", comme l'écrivait François Furet ${ }^{29}$, témoins et historiens s'étaient accordés sur l'idée simple qu'en 1914-1918, on avait été tué, mais on n'avait pas tué. Peu importe les passages de Erich Maria Remarque ou de témoignages parlant de violence interpersonnelle directe ${ }^{30}$, peu importe le scandale du J'ai tué (1918) de Blaise Cendrars. Dominait une image simple : celle des préparations d'artillerie lointaine, $\mathrm{du}$ " feu roulant " et dévastateur, des sorties ratées dans le no man's land où les soldats se faisaient faucher par le barrage du nid de mitrailleuses, le travelling latéral du film de Lewis Milestone (1930) tiré du best-seller de Remarque étant assez explicite de ce point de vue $^{31}$. Le plaidoyer de Stéphane Audoin-Rouzeau et Annette Becker invitant à Retrouver la guerre, au début des années 2000, s'est fondé sur l'idée d'interroger les violences interpersonnelles, notamment à partir de l'archéologie contemporaine et de la fabrication de matraques de tranchées ${ }^{32}$. Mort donnée au corps à corps il y avait eut, et même si elle était loin d'égaler les proportions de décès liés aux blessures violentes de l'artillerie, elle avait existé.

C'est peut-être l'aspect le plus problématique du jeu. En effet, la première mission qui s'intitule "Tempête d'acier » est un cas d'espèce. On peut d'abord noter que par rapport à la version allemande (Stahlgewitter) et la version originale en anglais (Storm of Steel), la traduction française passe totalement à côté de la référence à Ernst Jünger ${ }^{33}$ et son roman célèbre Orages d'acier de 1920, mais il ne s'agit que d'un détail. La première séquence est conçue expressément pour plonger le joueur dans un torrent de violences. Le joueur qui

29 François Furet, Le Passé d’une illusion. Essai sur l'idée communiste au XXe siècle, Paris, Robert Laffont, Calmann-Lévy, 1995, p. 53.

30 Erich Maria Remarque, op. cit., chapitre IX.

31 Lewis Milestone, À l'Ouest, rien de nouveau, 131 minutes, 1930. La scène se situe à la $30^{\mathrm{e}}$ minute du film.

32 Stéphane Audoin-Rouzeau, Annette Becker, 14-18, retrouver la Guerre, Paris, Gallimard, Folio Histoire, 2000, p. 61-71 et notamment page 65.

33 Ernst Jünger, Orages d'acier. Journal de guerre, traduit de l'allemand par Henri Plard, Paris, Christian Bourgois, 1970 [1920]. 
est donc, rappelons-le, en vue à la première personne ${ }^{34}$ dans le FPS, meure à plusieurs reprises en essayant de se débattre à travers plusieurs tableaux, dont on sent que chacun met en place une saynète de violence technologique spécifique. Dans cette séquence de moins de quinze minutes, qui débute sur un cauchemar post-traumatique de soldat, on peut voir une liste impressionnante, un condensé de toutes les armes de la Grande Guerre, sans grand égard pour la chronologie : chars, mitrailleuses, Gewehr 98, barbelés, grenades, dirigeables, lance-flammes, gaz de guerre, artillerie... L'impression donnée, là encore, n'est pas fausse, et dit quelque chose de la rupture technologique ${ }^{35}$ et humaine qu'a pu représenter la Première Guerre mondiale par rapport aux conflits précédents ${ }^{36}$. Mais dans le même temps, les toutes premières images donnent à voir un combat au corps à corps de dizaines de soldats, emportés par la rage, qui se massacrent littéralement à coup de matraques de tranchées et de pelles de terrassement. Un soldat empale son ennemi avec sa baïonnette et il existe même une option, pour le joueur, pour charger à la baïonnette ${ }^{37}$. Un homme assène des coups de poing au visage à un autre homme à terre dans une agression à mains nues. Cette séquence vise à peindre l'enfer des tranchées. Et cependant, elle est largement exagérée. Elle montre des formes de violence qui ont existé, évidemment - on ne peut pas balayer d'un revers de la main, avec ce qui a été dit au-dessus sur la camaraderie, les moments où les soldats montaient en première ligne avec la haine au ventre face à des ennemis qui venaient de tuer leurs plus proches camarades. Mais ce sont des cas limites. Le corps à corps, le moment où la transgression des normes du temps de paix est la plus forte, était un moment rare, paroxystique. La littérature de témoignage montre à quel point ces pratiques ont représenté des traumatismes pour les auteurs. Le jeu, d'une certaine manière, s'il montre bien la guerre industrielle, cède totalement face aux impératifs du gameplay et au divertissement : à plusieurs reprises, le joueur peut ainsi assassiner de très près les soldats adverses, dans la première mission comme dans les suivantes.

34 De là à en déduire qu'il est en immersion dans l'expérience vidéoludique, il n'y a qu'un pas, qu'il n'est pourtant pas nécessaire de toujours franchir. Olivier Caïra, "L'expérience fictionnelle : de l'engagement à l'immersion ", in Bernard Guelton (éd.), Les Figures de l'immersion, Rennes, PUR, 2014, p. 61-76.

35 Stéphane Audoin-Rouzeau, Annette Becker, 14-18, op. cit., p. 40-42, ici p. 41 : " [...] les formes de la mort ont en un siècle considérablement changé : au début du XIX ${ }^{e}$ siècle, en temps de guerre, la maladie tue bien plus que le combat".

36 Les conflits précédents (guerre napoléonienne, guerre de Crimée, guerre de Sécession) sont plus modernes qu'on a longtemps voulu le croire, du point de vue des seuils de violence et des " ruptures techniques », mises en valeur par les théoriciens militaires dès les années 1830. Voir Anne Rasmussen, "Blessures et blessés ", in Bruno Cabanes (éd.), Une histoire de la Guerre du XIX'e siècle à nos jours, Paris, Seuil, 2018, p. 438-452. Cependant, la Grande Guerre représente une "croissance exponentielle ", avec $30 \%$ des mobilisés blessés, sans que ce chiffre prenne en compte les blessures cumulées. Ibid., p. 445.

37 Cédric Marty, À l'assaut! La baïonnette dans la Première Guerre mondiale, Paris, Vendémiaire, 2018. 
L'historien s'efface ici devant le game designer: la fiction du jeu réaliste s'évapore au profit du "plaisir ludique " $\left(\right.$ playsir $\left.^{38}\right)$, preuve du mélange des genres toujours présent dans ces produits culturels.

Cela peut donner l'impression que la Grande Guerre a été une orgie de destruction individuelle; or elle a bien été une orgie de destruction, mais bien souvent collective. Et le jeu, de ce point de vue, repose sur un soldat toujours très autonome, qui reçoit peu d'ordres et agit seul, ou en très petites unités. Ce faisant, le gameplay s'approche bien plus d'une représentation des unités de troupes d'assaut qui se développent à la toute fin de la guerre que de la réalité d'un régiment d'infanterie de 1914 ou de 1915. De ce point de vue, la critique revient en permanence dans les débats des joueurs, beaucoup louant le réalisme bien plus profond du jeu Verdun (M2H/Blackmill Games, 2015), que ce soit dans cette peinture de la violence, dans le niveau de difficulté ou dans les types de stratégie mis en place pour combattre.

\section{Conclusion}

Nous n'avons ici qu'effleuré quelques thématiques soulevées par le jeu Battlefield 1. Il en reste beaucoup d'autres à approfondir, que ce soit en analysant les interactions dans le mode multijoueurs, ou en prenant également en compte une foule de détails que nous n'avons pas pu aborder ici, de la présence des animaux dans le jeu (notamment les pigeons), très en phase avec les dernières découvertes historiographiques ${ }^{39}$, à la question des relations interpersonnelles, ou à la construction de la virilité militaire dans le jeu. Il est tout aussi intéressant de confronter ce divertissement aux faits historiques - dans une quête de vérité - que de l'analyser en soi, comme produit culturel sur la Grande Guerre. De ce point de vue, il sculpte - bien au-delà de l'amusement produit par le jeu - une certaine mémoire de la Première Guerre mondiale, en la transmettant aux jeunes générations, n’hésitant pas à dénoncer la guerre de 14-18 comme une boucherie dont le sens n'était pas directement évident aux soldats. Pour toutes ces raisons, il mérite l'attention des historiens.

\section{Nicolas Patin}

Maître de conférences en histoire contemporaine Université Bordeaux Montaigne nicolas.patin@u-bordeaux-montaigne.fr

38 Stéphane Vial, L'Être et l'écran. Comment le numérique change la perception, Paris, PUF, 2013, p. 246 : "C'est ludique parce que c'est source de plaisir, un plaisir jouable que nous appelons à dessein un "playsir", car il existe une essence commune entre plaisir et jeu ".

39 Éric Baratay, Bêtes de tranchées : des vécus oubliés, Paris, CNRS Éditions, 2013 ; Claude Milhaud, 1914-1918. L'autre hécatombe. Enquête sur la perte de 1140000 chevaux et mulets, Paris, Belin, 2017. 
Nicolas Patin est un ancien élève de l'École normale supérieure de Lyon-LSH, agrégé et maitre de conférences en histoire contemporaine à l'Université Bordeaux Montaigne, il est spécialiste de l'Allemagne du premier $\mathrm{XX}^{\mathrm{e}}$ siècle, de la Première Guerre mondiale et du national-socialisme. Sans être un joueur assidu, il s'intéresse aux contenus historiques des jeux vidéo, quand ceux-ci rentrent dans ses champs de compétences. Il a organisé en 2018 et 2019 plusieurs séances interactives durant lesquelles un historien commentait des séquences de jeux joués en direct, à l'Université Bordeaux Montaigne.

\title{
Résumé
}

Première Guerre mondiale et jeux vidéo sont rarement associés dans l'industrie vidéoludique et les historiens ne s'intéressent par ailleurs qu'assez peu aux jeux. Le jeu Battlefield 1 (DICE/ EA) a pourtant été l'événement du Centenaire, avec des millions d'exemplaires vendus à travers le monde. Quel intérêt y a-t-il à en faire une analyse historique ? Le jeu est en lui-même une production culturelle à propos de la Grande Guerre. Plus encore, il sait parfois donner à voir des vérités à la pointe des débats historiographiques, tout comme faire de grossières erreurs.

\section{Mots-clés}

Première Guerre mondiale, Jeu vidéo, Battlefield, Violence de guerre, Gameplay.

\begin{abstract}
World War I and video games are rarely associated in the video game industry and historians have showed little interest in video games. However, the game Battlefield 1 (DICE/EA) was the major event of the Centenary, with millions of copies sold worldwide. What can be learned through an historical analysis of this game? Battlefield 1 itself is a cultural production about the Great War. Moreover, it sometimes shows facts, which are at the forefront of the historiographical debat, as well as making serious mistakes.
\end{abstract}

\section{Keywords}

First World War, Video game, Battlefield, War violence, Gameplay. 